\title{
Development of Attic Cholesteatoma in Acute Otitis Media with Tuberculosis
}

\author{
Generoso T. Abes, ${ }^{1}$ Franco Louie LB Abes ${ }^{2}$ and Teresa Luisa G. Cruz ${ }^{1}$ \\ ${ }^{1}$ Department of Otorhinolaryngology, College of Medicine and Philippine General Hospital, University of the Philippines Manila \\ ${ }^{2}$ College of Medicine, University of Santo Tomas
}

\begin{abstract}
Literature has reported the existence of cholesteatoma in chronic suppurative otitis media with tuberculosis. This report demonstrates that a cholesteatoma can be associated with an acute otitis media with tuberculosis. The importance of a high index of suspicion for tuberculous otitis media and its consequent meticulous management including diagnostic tests, medical and surgical procedures and monitoring are discussed.
\end{abstract}

Key Words: tuberculous otitis media, TB and cholesteatoma, tuberculosis of the ear

\section{Introduction}

Aural cholesteatoma is a common complication of chronic otitis media ${ }^{1}$ but only few cases were reported in association with tuberculosis otitis media (TBOM). ${ }^{2-4}$ TBOM itself is rare ${ }^{5,6}$ and cholesteatoma in association with TBOM is even more infrequent. ${ }^{3}$ The coexistence of these two disease entities is believed to be a mere coincidence. ${ }^{3}$ Moreover, in a report on six cases of chronic otitis media with cholesteatoma formation, it was concluded that the presence of tuberculosis (TB) was just a secondary infection. ${ }^{7}$ Theories on the pathophysiologic routes of TBOM include hematogenous, direct extension from the upper respiratory tract via the Eustachian tube, or by direct implantation through the external ear canal. ${ }^{4-6}$ Cholesteatoma, on the other hand, is a non-cancerous abnormal growth of keratinizing squamous epithelium in the middle ear and/or mastoid that commonly develops in chronic otitis media $(\mathrm{COM})^{8-9}$ It can be acquired or congenital. The exact etiopathogenesis of acquired cholesteatoma for every case is unclear ${ }^{10}$, and many otologists believe a hybrid process of four mechanisms (retraction pocket, migration, squamous metaplasia, basal cell hyperplasia) account for many cases. ${ }^{9}$ Because the

Corresponding author: Teresa Luisa G. Cruz, MD, MHPEd

Department of Otorhinolaryngology

2nd Floor, Ward 10

Philippine General Hospital

University of the Philippines Manila

Taft Avenue, Ermita, Manila 1000 Philippines

Telephone: +6325548400 local 2151 or 2152

Email: techgloriacruz@yahoo.com coexistence of cholesteatoma with TB infection was almost exclusively found in cases presenting with eardrum perforation, the theory of TB bacilli implantation via the external ear canal is often considered as the likely possible etiologic mechanism of TB infection. ${ }^{2-3}$ This report describes the occurrence of cholesteatoma in a new-onset otitis media, which also had tuberculous infection.

\section{Case Report}

An 18-year-old generally healthy male student consulted for the management of attic cholesteatoma, right ear. Four years prior to consult, he developed right middle ear effusion and was diagnosed to have Acute Otitis Media (AOM), Right ear. His left ear at this time was asymptomatic by history and normal on examination. He was treated with several oral antibiotics, oral steroid, and other adjunct treatments for $\mathrm{AOM}$ but treatment failed to relieve the symptoms. Myringotomy with ventilation tube insertion was done, with the VT extruded after 1 year, and with persistence of hearing loss. Three years later he was diagnosed with attic cholesteatoma on the same right ear based on examination. An audiogram showed right mild to moderate conductive hearing loss. The right ear temporal bone CT showed soft tissue densities in the attic and mastoid, well-preserved mastoid air cell architecture, mastoid bone sclerosis, absent scutum erosion, and intact ossicles (Figure 1). By contrast, the left ear history, otoscopy, audiogram, and CT images were all normal except for sclerotic mastoid bone (Figure 1). His past history was positive for sinusitis and he was given treatment for primary tuberculosis (TB) at age eight years old.

A planned single stage canal down surgery with fascia graft tympanoplasty, cortical bone ossiculoplasty and cartilage mastoid obliteration was done on the right ear. Three months after surgery the tympanic membrane graft appeared intact but thickened (Figure 2a). Part of the fascia graft laid over the mastoid cavity remained swollen, congested, and wet due to recurrent serous discharge (Figure 2b). Quinolone ototopical solution with or without steroid, occasionally combined with various oral antibiotics, failed to provide lasting relief. A repeat audiogram three months after surgery showed persistent conductive hearing loss on the right ear and still normal hearing thresholds on the left ear. 

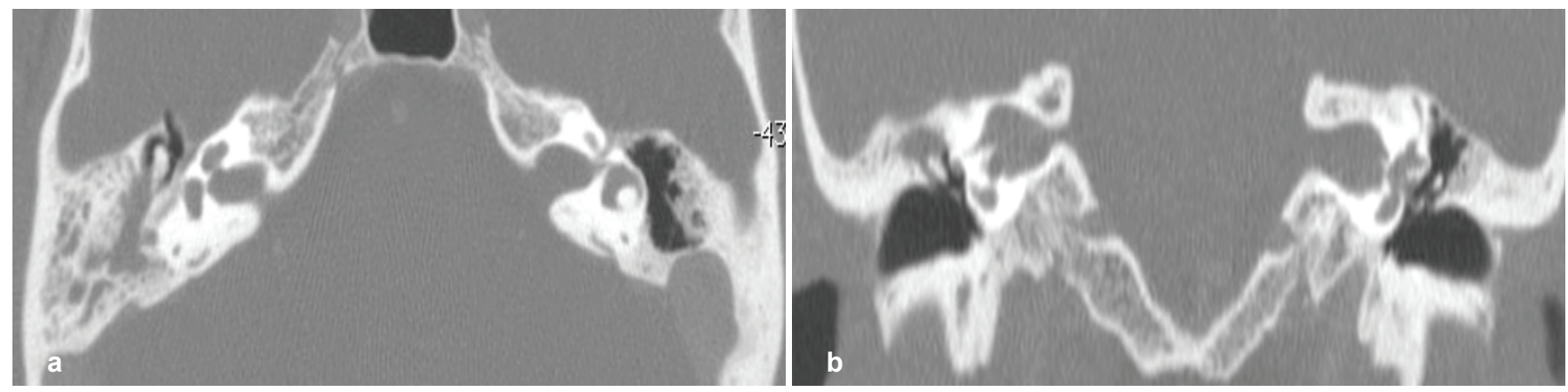

Figure 1. Temporal bone CT one month prior to right ear surgery, showing haziness over the attic and mastoid, compatible with otoscopic presence of attic cholesteatoma. Note the normal left temporal bone, compatible with normal otoscopic findings.
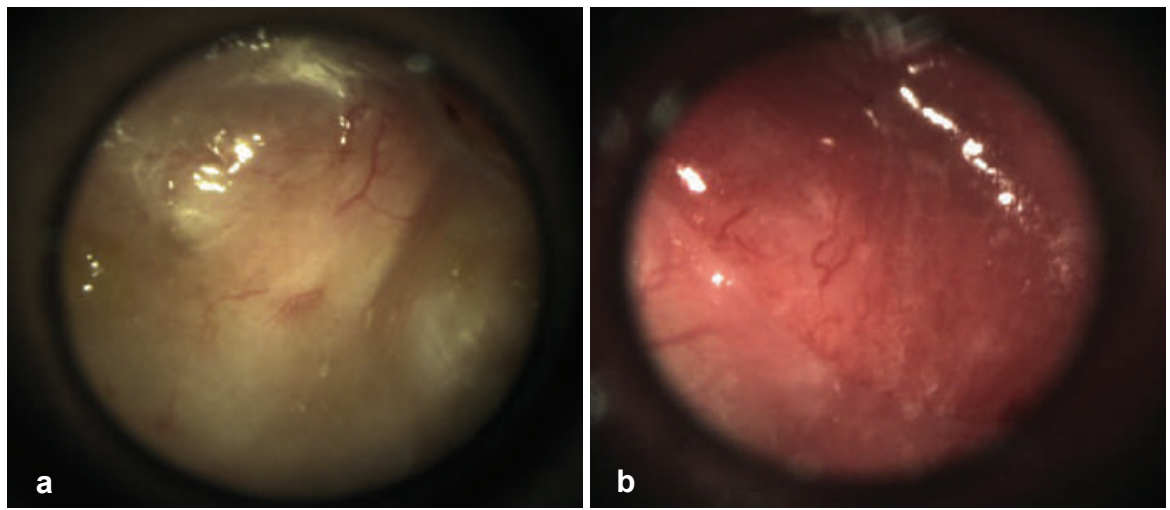

Figure 2. Postoperative otoscopic appearance of the fascia graft laid over the right middle ear and mastoid cavities, showing the thickened, draining eardrum and mastoid graft.
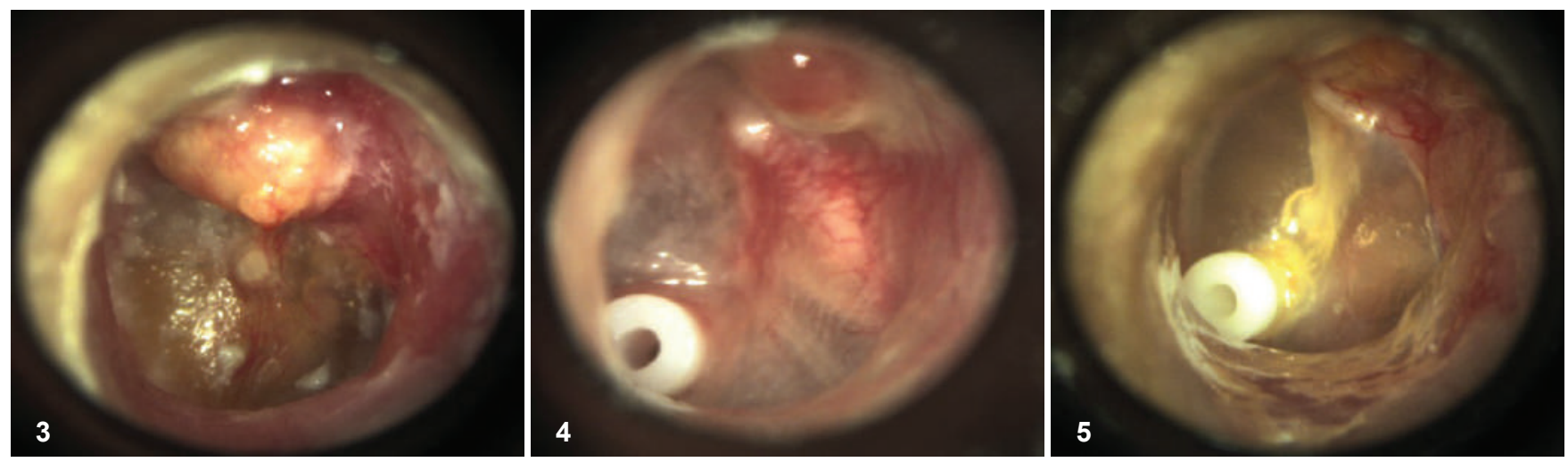

Figure 3. Otoscopy of the left ear, three years after the right ear surgery. Figure 4. Otoscopy of the left ear, one month after myringotomy, showing attic granulation tissue and partial resolution of middle ear fluid. Figure 5. Otoscopy of the left ear after completion of six-month anti-TB treatment, showing thin eardrum, unremarkable attic and complete resolution of eardrum congestion and middle ear fluid.

Three years later, the patient complained of otalgia, fever and hearing loss, this time, in the contralateral, previously normal, LEFT ear. The left eardrum appeared dull and congested with a yellowish mass over the pars flaccida (Figure 3). He was given a two week course of oral amoxicillin plus clavulanic acid, then another one week course of oral levofloxacin with improvement of otalgia but persistent hearing loss. The RIGHT, previously operated ear, on the other hand, exhibited granular appearance on the persistently draining mastoid cavity. By this time, a tuberculin skin test (TST) was done and was positive with $20 \mathrm{~mm}$ induration. The chest radiograph showed normal findings. 

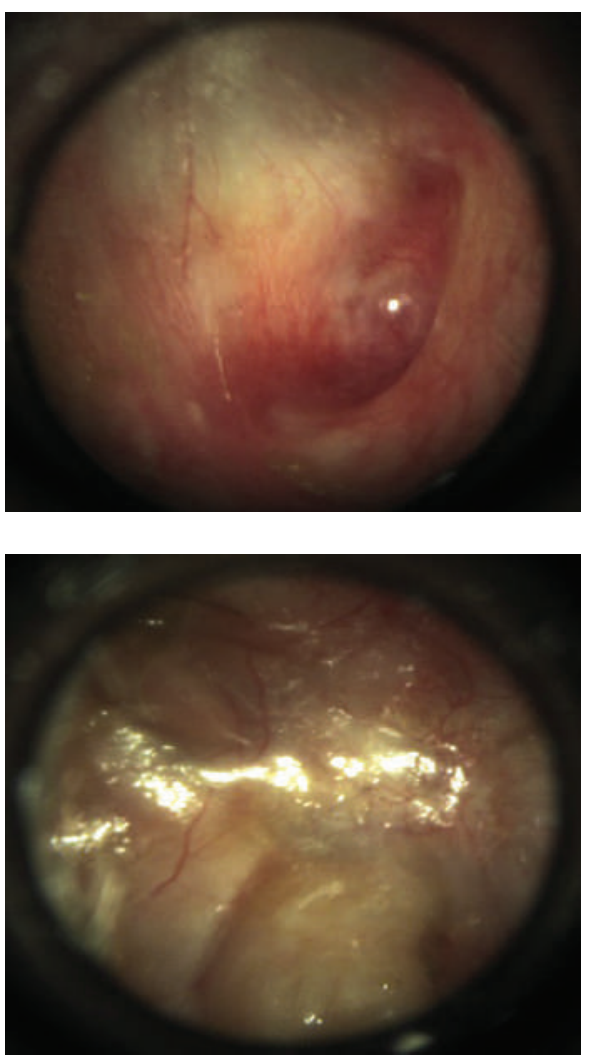

Figure 6. Otoscopy, right ear, after completion of 6-month anti-TB treatment, showing dryness and less thickening of eardrum graft and mastoid fascia graft.

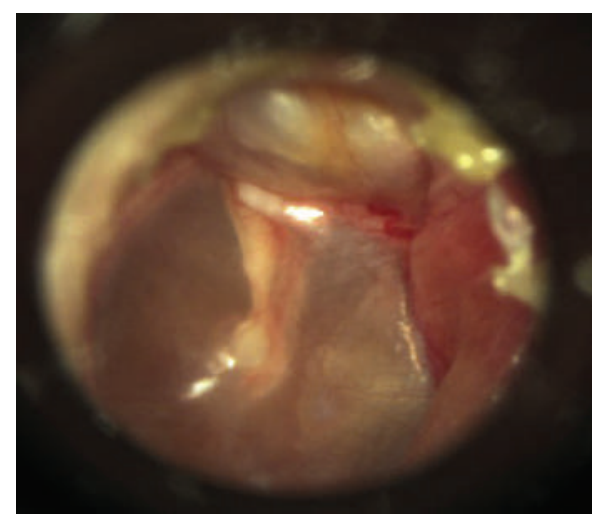

Figure 7. Otoscopy, left ear, showing the beginning attic cholesteatoma formation a year after completion of anti-TB medical therapy.

For the LEFT ear, Myringotomy, middle ear lavage and fluid aspiration, and ventilation tube insertion were done under local anesthesia. The collected aspirate was sent for Mycobacterium tuberculosis (MTb) polymerase chain reaction (PCR) study, with POSITIVE result. The attic mass was biopsied and also sent for PCR study, with positive result for cholesteatoma but negative for PCR. The histopathologic examination of the mass was positive for
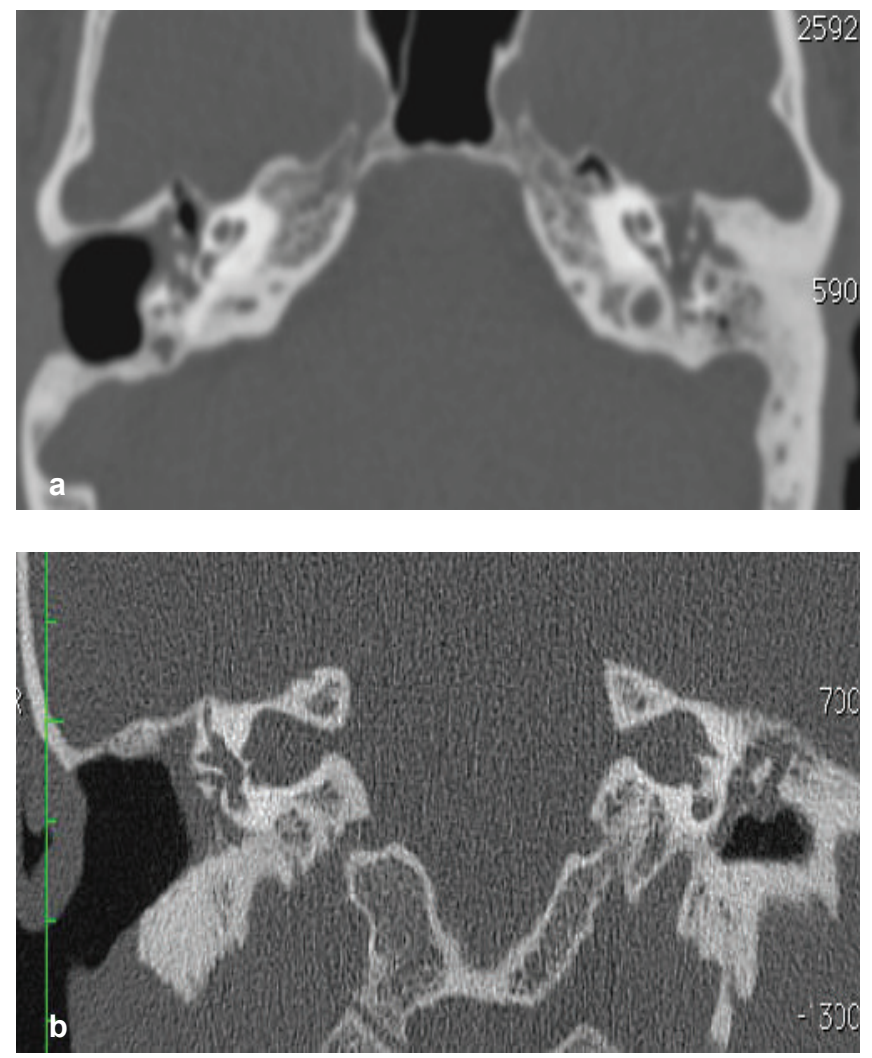

Figure 8. Repeat CT of temporal bone, four years after right ear surgery, showing attic, middle ear and mastoid densities at the time when the attic cholesteatoma was noted, 2014.

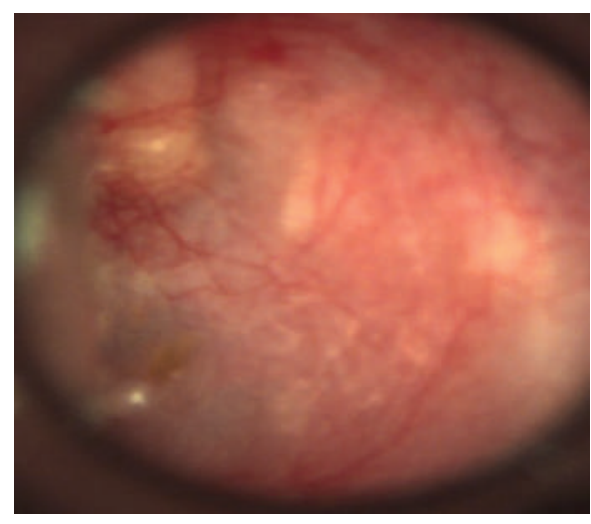

Figure 9. Otoscopic picture six months after surgery showing an intact well-healed eardrum graft, left ear.

cholesteatoma but negative for PCR M.Tb. A four-drug oral anti-TB combination consisting of isoniazid, rifampicin, ethambutol and pyrazinamide was given for two months, followed by a three-drug regimen for four months. There was resolution of middle ear inflammation and middle ear fluid, though granulation tissue remained in the attic area after the completion of the six month anti-TB medical therapy (Figures 4 and 5). The conductive hearing loss persisted on BOTH 
ears. Surprisingly, the previously draining post-operative RIGHT eardrum and mastoid fascia graft dried up without any oral or local medication (Figure 6). Lacking an explanation for the persistent hearing loss, serial audiometric tests were done until the ventilation tube on the LEFT ear was extruded a year later. At this time, an evident sign of attic cholesteatoma was observed (Figure 7). A repeat temporal bone CT showed opacities over the LEFT ear pars flaccida with scutum erosion and diffuse middle ear and mastoid cavities (Figure 8), whereas there were no radiographic findings of attic cholesteatoma in the CT images four years earlier (Figure 1). The patient thus underwent surgery for the LEFT ear attic cholesteatoma. During surgery, the presence of attic and mastoid cholesteatoma was confirmed on the left ear. The otoscopic findings six months after surgery on the left ear are shown in Figure 9.

\section{Discussion}

The case discussed presents clinical evidence that attic cholesteatoma may arise in concurrence with acute otitis media with TB infection. This contradicts a conclusion in another study stating that $\mathrm{TB}$ infection is secondary to chronic otitis media with cholesteatoma formation. ${ }^{7}$ Animal studies on cholesteatoma pathogenesis confirmed the induction of papillary projections of the pars flaccida basal layer into the attic space to form cholesteatoma in the presence of acute middle ear inflammatory process, whether infectious or non-infectious in character. ${ }^{9-10} \mathrm{We}$ believe this theory favors the development of attic cholestetoma in this case.

On the other hand, although the alternative etiology that an attic retraction pocket paved the way for cholesteatoma development exists, an intact epithelium over the attic mass (Figure 3) refutes this hypothesis. Still, we could not totally exclude the possibility of micro-retraction pocket that was not detected by video-otoscopy or intraoperative findings. Whatever the cause may be, our observation negates the belief that the presence of cholesteatoma in aural TB is a mere coincidence. Further, it provides evidence that TBOM is not only found in chronic middle ear infection as observed in many studies. ${ }^{4,6,11}$ It could also be detected in acute OM presenting with middle ear effusion, as observed in reports. ${ }^{2,4,12}$ It may then be hypothesized that atypical clinical presentation taken in conjunction with the failure of OM resolution after optimal antimicrobial treatment must alert the clinician to the possibility of TB infection in all otitis media cases- both acute and chronic processes.

Unresolved clinical signs of acute OM despite of adequate antimicrobial treatment suggests a complicated disease. ${ }^{14}$ The patient's persistent middle ear effusion with concurrent attic mass was an atypical feature of bacterial, non-TB acute OM. The acute onset of moderate mixed hearing loss in the previously normal hearing ear could be due to unrestricted inflammatory products that could had damaged host tissues in terms of middle ear scarring and inner ear toxicity. Although these findings could be observed both in non-TB and TB otitis media, ${ }^{13,14}$ the unexplained presence of the other abovementioned clinical features favored TBOM, rather than non-TBOM. On the other hand, radiologic features including diffuse middle ear and mastoid haziness, preserved mastoid air cell architecture, and absent bone erosions (Figure 8) in a previously well-aerated temporal bone (Figure 1), though probably not specific to TBOM, provided additional evidences that TB infection could indeed be detected in acute middle ear disease. ${ }^{2}$ Thus, these atypical disease features could be important clues to pursue the appropriate diagnostic tests.

The persistent moderate conductive hearing loss in spite of completely resolved eardrum inflammation affirmed the recommendation that more complicated disease deserves follow-up assessments.5,11 As exemplified by our case, it could reveal an uncommon complex clinical entity, like the development of attic cholesteatoma, in consonance with otitis media effusion (Figure 7). Adopting the attitude of close surveillance is important because an undetected complicated TBOM is associated with less favorable treatment results. ${ }^{12}$ Our case, nevertheless, showed that even in a complex middle ear disease, the appropriate surgical measure for cholesteatoma could still be done in a standard way to eliminate the pathology, reconstruct the middle ear mechanism, and improve hearing.

In summary, our study demonstrated that cholesteatoma could develop in acute otitis media with TB infection. Although its pathophysiology is conjectural, it typifies the difficulty and dilemma ${ }^{4,5,11}$ encountered in TBOM diagnosis. There is a need to discover other atypical forms of TBOM in order to deepen our understanding in managing this rare, yet challenging, middle ear condition.

\section{References}

1. Cunningham M, Guardiani E, Kim HJ, Brook I. Otitis media. Future Microbiol. 2012; 7(6):733-53.

2. Yaniv E. Tuberculous otitis media: a clinical record. Laryngoscope. 1987; 97(11):1303-6.

3. Ishak NL, Zaina A, Abdullah A. Cholesteatoma and tuberculosis: Association or coincidence. Brunei Int Med J. 2012; 8(5):275-8.

4. Nishiike S, Irifune M, Doi K, Osaki Y, Kiuchi N. Tuberculous otitis media: Clinical aspects of 12 cases. Ann Otol Rhinol Laryngol. 2003;112(11):935-8.

5. Dale OT, Clarke AR, Drysdale AJ. Challenges encountered in the diagnosis of tuberculous otitis media: case report and literature review. J Laryngol Otol. 2011; 125(7):738-40.

6. Windle-Taylor PC, Bailey CM. Tuberculous otitis media: a series of 22 patients. Laryngoscope. 1980; 90(6Pt1):1039-44.

7. Yaniv E. Tuberculous otitis media as a secondary infection to chronic otitis media with cholesteatoma. Am J Otol. 1987; 8(1):40-2.

8. Olszewska E, Rutkowska J, Ozgirgin N. Consensus-Based Recommendations on the Definition and Classification of Cholesteatoma. J Int Adv Otol. 2015;11(1):81-7. 
9. Kuo $\mathrm{C}$, Shiao AS, Yung $\mathrm{M}$, et al. Updates and knowledge gaps in cholesteatoma research. 2015; 2015:854024.

10. Yamamoto-Fukuda T, Takahashi H, Koji T. Animal models of middle ear cholesteatoma. J Biomed Biotechnol. 2011; 2011:394241.

11. Robertson K, Kumar A. Atypical presentations of aural tuberculosis. Am J Otoalryngol. 1995; 16(5):294-302.

12. Abes GT, Abes FL, Jamir JC. The variable clinical presentation of tuberculosis otitis media and the importance of early detection. Otol Neurotol. 2011; 32(4):539-43.
13. Ahmad S. Pathogenesis, immunology, and diagnosis of latent Mycobacterium tuberculosis infection. Clin Dev Immunol. 2011; 2011:814943.

14. Leichtle A, Lai Y, Wollenberg B, Wasserman SI, Ryan AF. Innate signaling in otitis media: pathogenesis and recovery. Curr Allergy Asthma Rep. 2011;11(1):78-84.

\section{The}

Nationall ilealth science Journal

\author{
is now indexed in
}

\section{Sciverse Scojpus.}

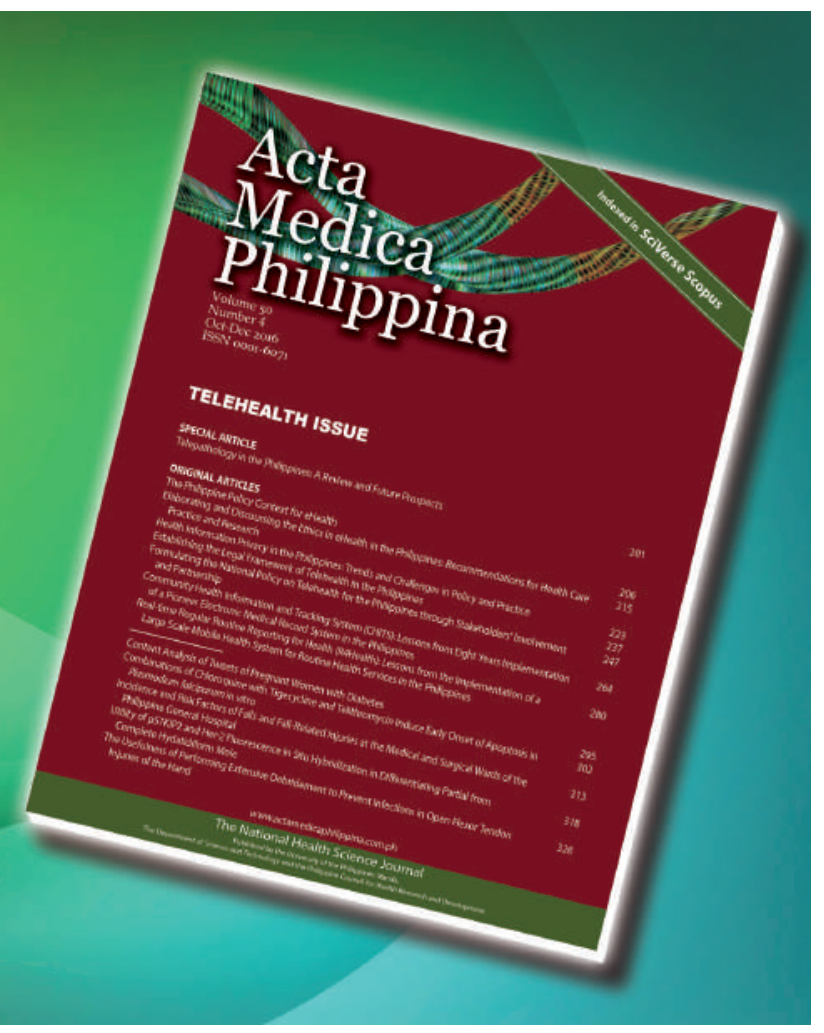

